Pacific Journal of Mathematics

CHAINS OF MODULES WITH COMPLETELY REDUCIBLe 


\title{
CHAINS OF MODULES WITH COMPLETELY REDUCIBLE QUOTIENTS
}

\author{
JOHN DAUNS
}

Consider a left module $V$ over a possibly noncommutative ring $R$. The objective is to investigate finite or infinite sequences of submodules of $\boldsymbol{V}$ of the form $\{0\}=A_{0} \subseteq A_{1} \subseteq A_{2} \cdots$ or of the form $V=A^{0} \supseteq A^{1} \supseteqq \dot{A}^{2} \cdots$ where all the quotient modules $A_{i+1} / A_{i}$ or $A^{i} / A^{i+1}$ are completely reducible. It is shown that some of the known properties of such series for a module over a ring with minimum condition hold for a more general class of rings, a class which properly includes those satisfying the descending chain condition. The main difficulty which this note has attempted to solve is to generalize these well known theorems from the minimum condition case to a much larger class of rings and modules. The class of rings considered in this note seems to be the natural setting in which to prove these theorems. In spite of the added generality, our proofs are not longer than they would be if the minimum condition were assumed.

All modules considered here will be understood to be left modules. A module $\boldsymbol{V}$ over a ring will be called simple provided it contains no proper nonzero submodules and provided also $\boldsymbol{R} \boldsymbol{V}=\boldsymbol{V} \neq\{0\}$. A module is completely reducible provided it is a finite or infinite direct algebraic sum of simple modules.

In the next definitions and subsequently, the set inclusion symbol " $\subset$ " will always indicate a proper inclusion. The next two definitions are essentially taken from [4, p. 103].

Definition. Suppose $\boldsymbol{V}$ is any left module over an arbitrary ring $\boldsymbol{R}$. Define $\boldsymbol{L}_{0}(\boldsymbol{V})$ to be the zero module. For any positive integer $k=1,2, \cdots$ let $L_{k}(V)$ be defined inductively as the algebraic sum of all submodules $\boldsymbol{Y}$ of $\boldsymbol{V}$ with $\boldsymbol{L}_{k^{-1}}(\boldsymbol{V}) \subset \boldsymbol{Y}$ and with a simple quotient $\boldsymbol{Y} / \boldsymbol{L}_{\boldsymbol{k}-1}(\boldsymbol{V})$. If $L_{\alpha}(V)$ has been defined for all $\alpha<\beta$; where $\beta$ is a limit ordinal, set $\boldsymbol{L}_{\boldsymbol{\beta}}(\boldsymbol{V})=\cup\left\{\boldsymbol{L}_{\alpha}(\boldsymbol{V}) \mid \alpha<\beta\right\}$ and define $\boldsymbol{L}_{\beta+1}(\boldsymbol{V})$ to be the sum of all submodules $\boldsymbol{Y}$ of $\boldsymbol{V}$ with $\boldsymbol{L}_{\beta}(\boldsymbol{V}) \subset \boldsymbol{Y}$ and with a simple quotient $\boldsymbol{Y} / \boldsymbol{L}_{\boldsymbol{\beta}}(\boldsymbol{V})$. The empty sum is taken to be the zero module. The series of submodules $\{0\}=\boldsymbol{L}_{\hat{i}}(V) \subseteq \boldsymbol{L}_{1}(V) \subseteq \boldsymbol{L}_{i}(V) \subseteq \cdots$ is called the lower Loewy series of $\boldsymbol{V}$ over $\boldsymbol{R}$.

Definition. For a left module $\boldsymbol{V}$ over a ring $\boldsymbol{R}$, set $\boldsymbol{L}^{\mathrm{q}}(\boldsymbol{V})=\boldsymbol{V}$

Received January 6, 1965. This research was partially supported by N. S. F. Grant GP 1877. 
and denote by $\boldsymbol{L}^{1}(\boldsymbol{V})$ the intersection of all the maximal proper submodules of $\boldsymbol{V}$. The empty intersection is by convention all of $\boldsymbol{V}$ and the zero module is a maximal submodule of a simple module. For any positive integer $k=1,2, \cdots$ the submodule $\boldsymbol{L}^{k}(\boldsymbol{V})$ is defined inductively by $L^{k}(V)=L^{1}\left[L^{k-1}(V)\right]$. If $\boldsymbol{L}(V)$ has been defined for all ordinals $\alpha<\beta$ where $\beta$ is a limit ordinal, set $\boldsymbol{L}^{\beta}(\boldsymbol{V})=U\left\{\boldsymbol{L}^{\alpha}(\boldsymbol{V}) \mid \alpha<\beta\right\}$ and define $\boldsymbol{L}^{\beta+1}(\boldsymbol{V})$ to be $L^{\beta+1}(\boldsymbol{V})=L^{1}\left[\boldsymbol{L}^{\beta}(\boldsymbol{V})\right]$. The series $\boldsymbol{V}=\boldsymbol{L}^{0}(\boldsymbol{V}) \supseteqq$ $\boldsymbol{L}^{1}(\boldsymbol{V}) \supseteqq \boldsymbol{L}^{2}(\boldsymbol{V}) \supseteqq \cdots$ is called the upper Loewy series of $\boldsymbol{V}$ over $\boldsymbol{R}$.

Clearly, the quotients of the consecutive terms of the lower Loewy series are completely reducible; it will be shown later that this also holds for the upper series for all superscripts. It will also be shown that, in a sense which is more precisely defined in conclusions 3 of the Theorems 2 and 4, the lower and the upper Loewy series are the unique biggest chains of submodules with completely reducible quotients. The indices on the terms of the lower and upper Loewy series will henceforth be positive integers; except in the example at the very end, and even here they will only be $\omega$ and $\omega+1$, where $\omega$ is the first infinite ordinal.

The radical of any ring $\boldsymbol{R}$, denoted by $\boldsymbol{N}$, will be the usual Jacobson radical, i.e., the intersection of the annihilators of all simple left $\boldsymbol{R}$-modules $\boldsymbol{U}$ with $\boldsymbol{R} \boldsymbol{U}=\boldsymbol{U}$. In case there do not exist any simple $\boldsymbol{R}$-modules, $\boldsymbol{N}=\boldsymbol{R}$.

From now on it will be assumed that the module $\boldsymbol{V}$ satisfies the condition that

(1) for any submodule $\boldsymbol{U}$ of $\boldsymbol{V}, \boldsymbol{R} \boldsymbol{U}=\boldsymbol{U} \cdot$ A restriction will be, imposed on the ring by requiring that

(2) $\boldsymbol{R} / \boldsymbol{N}$ is completely reducible, i.e., either zero or a direct sum of minimal left ideals $\overline{\boldsymbol{L}}$ with $\boldsymbol{R} \overline{\boldsymbol{L}}=\overline{\boldsymbol{L}}$. The ring $\boldsymbol{R}$ considered here is neither assumed to be commutative nor to contain a unity element. Indeed, one of our objectives has been to develop the properties of the two Loewy series so that they would be applicable to rings which cannot in principle contain a unity element, e.g., if $\boldsymbol{R} / \boldsymbol{N}$ is the ring of all linear transformations with finite dimensional ranges on an infinite dimensional vector space. In the latter example, clearly (1) and (2) are satisfied.

2. The lower Loewy series. The next lemma will be used frequently.

LEMMA 1. Let $\boldsymbol{R}$ be a completely reducible ring with zero radical. Then any left $\boldsymbol{R}$-module $\boldsymbol{U}$ with $\boldsymbol{R} \boldsymbol{U}=\boldsymbol{U}$ is completely reducible.

Proof. The ring $\boldsymbol{R}$ is the algebraic direct sum $\boldsymbol{R}=\bigoplus\left\{\boldsymbol{L}_{\boldsymbol{i}} \mid i \in I\right\}$ 
where $\boldsymbol{L}_{i}$ is a minimal left ideal of $\boldsymbol{R}$ with $\boldsymbol{R} \boldsymbol{L}_{\boldsymbol{i}}=\boldsymbol{L}_{\boldsymbol{i}} \neq\{0\}$, and $I$ is some indexing set. For any $v \in \boldsymbol{U}, v$ is of the form $v=r_{1} w_{1}+\cdots$ $+r_{n} w_{n}$ where $r_{k} \in \boldsymbol{L}_{\boldsymbol{i}(\boldsymbol{k})}$ and $w_{k} \in \boldsymbol{U}$ for $i(k) \in I$ and $k=1,2, \cdots, n$. Let $\boldsymbol{L}$ be a minimal left ideal of $\boldsymbol{R}$ with $\boldsymbol{R} \boldsymbol{L}=\boldsymbol{L}$. It suffices to show that if for some $v \in \boldsymbol{U}$ we have $\boldsymbol{L} v \neq 0$, that then $\boldsymbol{L} v$ is a simple submodule of $\boldsymbol{U}$. But, if $W$ were a proper submodule of $\boldsymbol{L} v$, then $J=\{t \in \boldsymbol{L} \mid t v \in W\}$ would be a left ideal of $\boldsymbol{R}$ properly contained in $\boldsymbol{L}$.

CoRollaRY. Let $\boldsymbol{R}$ be a ring with radical $\boldsymbol{N}$ such that $\boldsymbol{R} / \boldsymbol{N}$ is completely reducible. Assume that $\boldsymbol{U}$ is any left $\boldsymbol{R}$-module with $\boldsymbol{R} \boldsymbol{U}=$ $\boldsymbol{U}$. Then $\boldsymbol{U}$ is completely reducible if and only if $\boldsymbol{N U}=\{0\}$.

Proof. If $\boldsymbol{U}$ is a sum of simple modules, then $\boldsymbol{N} \boldsymbol{U}=\{0\}$ because $\boldsymbol{N}$ annihilates any simple $\boldsymbol{R}$-module. Conversely, if $\boldsymbol{N} \boldsymbol{U}=\{0\}$, then $\boldsymbol{U}$ viewed as an $R / N$-module is completely reducible over $R / N$ and hence also over $\boldsymbol{R}$.

The next theorem is the main result about the lower Loewy series. For any subset $B$ of a ring $R$, the symbol $B^{\perp}$ denotes the subset of a fixed $\boldsymbol{R}$-module $\boldsymbol{V}$ which is annihilated by $B$.

THeOREM 2. Let the ring $\boldsymbol{R}$ with radical $\boldsymbol{N}$ and the module $\boldsymbol{V}$ be as in (1) and (2). Then the following holds for all integers $k=$ $0,1,2, \cdots$ :

1. $\boldsymbol{L}_{k+1}(\boldsymbol{V}) / \boldsymbol{L}_{\boldsymbol{k}}(\boldsymbol{V})$ is completely reducible.

2. $L_{k}(V)=N^{k \perp}$.

3. Uniqueness: if $\{0\}=A_{0} \subseteq A_{1} \subseteq A_{2} \subseteq \cdots$ is any series of submodules of $\boldsymbol{V}$ with completely reducible quotient modules $A_{k+1} / A_{k}$ for $k=0,1,2, \cdots$, then $A_{k} \leqq L_{k}(V)$.

Proof. Conclusion 1 is clear.

Conclusion 2 is true for $k=0$. By definition $N^{0}=R$. If there exist elements $v \in \boldsymbol{V}, v \neq 0$, for which $\boldsymbol{R} v=0$, let $\boldsymbol{U}=\{v \in \boldsymbol{V} \mid \boldsymbol{R} v=0\}$. By (1), $\boldsymbol{R} U=\boldsymbol{U}$, a contradiction.

Assuming conclusion 2 to be true for $k-1$, i.e., $L_{k^{-1}}(V)=\left(N^{k-1}\right)^{\perp}$, we prove it for $k$. Let $\boldsymbol{U}$ be the completely reducible left $\boldsymbol{R}$-module $\boldsymbol{U}=\boldsymbol{L}_{k}(\boldsymbol{V}) / \boldsymbol{L}_{k-1}(\boldsymbol{V})$. Since $\boldsymbol{L}_{\boldsymbol{k}}(\boldsymbol{V})$ is a submodule of $\boldsymbol{V}$, by assumption (1) we have $R \boldsymbol{L}_{\boldsymbol{k}}(\boldsymbol{V})=\boldsymbol{L}_{\boldsymbol{k}}(\boldsymbol{V})$ and consequently $\boldsymbol{R} \boldsymbol{U}=\boldsymbol{U}$. Applying Lemma 1 to the module $U$, we find that $N L_{k}(V) \subseteq L_{k-1}(V)=\left(N^{k-1}\right)^{\perp}$ and hence that $L_{k}(V) \subseteq N^{k \perp}$. However, since $N N^{k \perp} \subseteq\left(N^{k-1}\right)^{\perp}$, Lemma 1 again guarantees that the module $N^{k \perp} /\left(N^{k-1}\right)^{\perp}$ is completely reducible, and hence we have the opposite inclusion $N^{k \perp} \cong L_{k}(V)$.

To establish conclusion 3 , assume $A_{k-1} \subseteq L_{k-1}(V)$; the latter holds for $k=1$ and 2 . The complete reducibility of the module $A_{k} / A_{k-1}$ 
together with Lemma 1 implies that $N A_{k} \subseteq A_{k-1}$ and hence that $N A_{k} \subseteq$ $\boldsymbol{L}_{k-\mathrm{i}}(V)$. Thus $N^{k} A_{k} \subseteq N^{k-1} \boldsymbol{L}_{k-\mathrm{i}}(\boldsymbol{V})=\{0\}$, or $A_{k} \subseteq N^{k \perp}=\boldsymbol{L}_{k}(V)$.

3. The upper Loewy series. The next lemma is false unless the restriction (2) from the introduction is imposed on the ring.

Lemma 3. Let $\boldsymbol{R}$ be a ring with radical $N$ such that $\boldsymbol{R} / \boldsymbol{N}$ is completely reducible. Let $\boldsymbol{U}$ be a left $\boldsymbol{R}$ module with $\boldsymbol{R} \boldsymbol{U}=\boldsymbol{U}$. Then for any $v \in \boldsymbol{U}$ with $v \notin \boldsymbol{N} \boldsymbol{U}$, there exists a maximal proper submodule $M$ of $\boldsymbol{U}$ with $\boldsymbol{N U} \subseteq M$, but $v \notin M$.

Proof. By Lemma 1, the quotient module $U / N U$ is a direct sum of simple modules. The vector $v+N U$ can be written as a unique finite sum of vectors each of which lies in a simple submodule of $\boldsymbol{U} / \boldsymbol{N U}$. Let $P$ be a submodule of $U$ with $N U \subseteq P$, with $P / N U$ simple, and such that $v+N \boldsymbol{U}$ has a nonzero component in $P / N U$. Then $\boldsymbol{U} / \boldsymbol{N U}$ is the direct sum

$$
\frac{U}{N U}=\frac{P}{N U} \oplus \frac{M}{N U}
$$

where $M$ is a submodule of $U$ with $N U \subseteq M$. Clearly, $v \notin M$. Since

$$
\frac{U}{M} \cong \frac{U / N U}{M / N U} \cong \frac{P}{N U},
$$

it follows that $U / M$ is simple and hence that $M$ is maximal in $U$.

Next a counterexample is given to show that the last lemma is false if the ring $R$ fails to satisfy (2). Let $R$ be any commutative integral domain with a unit in which every ideal is principal and with a quotient field $Q$ not equal to $\boldsymbol{R}$. The module $Q$ over $\boldsymbol{R}$ cannot contain a maximal proper submodule $M$. Otherwise, let $a / b \notin M$ with $a, b \in R$. Then $Q=M+\mathbb{R}(1 / b)$ and $1 / b^{2}=\mathrm{m}+n / b$ for some $m \in M, n \in \boldsymbol{R}$. Hence $1 / b=b m+n$ and $n \notin M$. Thus $\mathbb{R} \nsubseteq M$ and for some $c \in \mathbb{R}$ with $c \neq 1$ or 0 , we have $R \cap M=R c$. Since $Q=M+R(1 / c)$, there exists $m \in M$ and $s \in R$ with $1 / c^{3}=m+s / c$. Consequently, $1 / c=\left(m c^{2}+s c\right) \in M$, contradicting the fact that $\boldsymbol{R} \nsubseteq M$. If $\boldsymbol{R}$ has a finite number of primes, then the radical $N$ of $R$ is the principal ideal generated by the product of all the primes. In this case $N Q=Q$, and the previous lemma is not applicable. If $R$ has an infinite number of primes then $N$ is zero. Since $\boldsymbol{R}$ cannot contain a single minimal ideal the hypothesis (2) that $\boldsymbol{R} / \boldsymbol{N}$ is a direct sum of minimal left ideals fails.

The following theorem about the upper Loewy series is a perfect analogue of Theorem 2 for the lower series.

THEOREM 4. Let the ring $\boldsymbol{R}$ with radical $\boldsymbol{N}$ and the module $\boldsymbol{V}$ be 
as in (1) and (2). Then the following holds for all $k=0,1,2, \cdots$ :

1. $\boldsymbol{L}^{k}(\boldsymbol{V}) / \boldsymbol{L}^{k+1}(\boldsymbol{V})$ is completely reducible.

2. $L^{k}(V)=N^{k} V$.

3. Uniqueness: if $V=A^{0} \supseteqq A^{1} \supseteqq A^{2} \supseteqq \cdots$ is any series of submodules of $\boldsymbol{V}$ with completely reducible quotient modules $A^{k} / A^{k+1}$ for $k=0,1,2$, then $A^{k} \supseteqq \boldsymbol{L}^{k}(\boldsymbol{V})$.

Proof. By use of Lemma 1, conclusion 1 is an immediate consequence of conclusion 2 .

In order to prove conclusion 2 , assume by induction that $L^{k-1}(V)=$ $\boldsymbol{N}^{k-1} \boldsymbol{V}$; the latter holds if $k=1$, since $N^{0}=R$ by definition and $R \boldsymbol{V}=$ $\boldsymbol{V}$ by assumption. If $v \in L^{k-1}(\boldsymbol{V})$ but $v \notin N L^{k-1}(V)$, then by Lemma 3 the element $v \notin L^{1}\left[L^{k-1}(V)\right]=L^{k}(V)$. Hence $N^{k} V \supseteqq L^{k}(V)$. Conversely, if $Q$ is a maximal submodule of $L^{k-1}(V)$, then by Lemma $1, N L^{k-1}(V) \subseteq$ $Q$, and hence $N^{k} V \subseteq L^{k}(V)$. Thus $L^{k}(V)$ equals $N^{k} V$.

Conclusion 3 is valid for $k=0$, assume it for $k-1$, i.e., $L^{k-1}(V) \subseteq$ $A^{k-1}$. Since the module $A^{k-1} / A^{k}$ is completely reducible, we have $N A^{k-1} \subseteq$ $A^{k}$ and consequently that $\boldsymbol{L}^{k}(\boldsymbol{V})=N \boldsymbol{L}^{k-1}(\boldsymbol{V}) \subseteq N A^{k-1} \leqq A^{k}$.

REMARKs. (i) It is not clear whether the hypothesis in Theorems 2 and 4 that for any submodule $\boldsymbol{U}$ of $\boldsymbol{V}, \boldsymbol{R} \boldsymbol{U}=\boldsymbol{U}$ cannot be weakened to require only that $\boldsymbol{R} \boldsymbol{V}=\boldsymbol{V}$. In order to do this, perhaps some additional hypothesis may have to be placed on $R$, e.g., $R N=N$.

(ii) The associativity of multiplication in the ring $\boldsymbol{R}$ was never used. For example, all of the previous considerations remain valid for a Lie module over a Lie ring.

(iii) It seems reasonable to conjecture that continuous analogues of the theorems of this paper could be formulated in order to be applicable to the case where the ring $\boldsymbol{R}$ consists of bounded linear transformations on a Hilbert space $V$. Then one would have to consider chains of subspaces of the Hilbert space $\boldsymbol{V}$ such that the quotient of any two adjacent terms would be a Hilbert space direct sum of closed $\boldsymbol{R}$-invariant subspaces containing no proper closed $\boldsymbol{R}$-invariant subspaces. The Jacobson radical would have to be replaced by a possibly bigger ideal-the intersection of all closed regular maximal left ideals of $\boldsymbol{R}$. (If $\boldsymbol{R}$ were norm-closed and commutative, then any regular maximal ideal would be automatically closed.)

Next some corollaries of Theorems 2 and 4 are derived. In part 3 of the next lemma the ring $\boldsymbol{R}$ is viewed as a left $\boldsymbol{R}$-module over itself.

CoRollary 5. Let the ring $\boldsymbol{R}$ and the module $\boldsymbol{V}$ be as in (1) and (2) and let $W$ be any submodule of $\boldsymbol{V}$. For conclusion 3 assume in addition that for any left ideal $I$ of $\boldsymbol{R}, \boldsymbol{R I}=I$. Then for all $k=$ $0,1,2, \cdots$ the following hold:

$$
\boldsymbol{L}_{\boldsymbol{k}}(W)=W \cap \boldsymbol{L}_{\boldsymbol{k}}(\boldsymbol{V})
$$




$$
\begin{gathered}
\boldsymbol{L}^{\boldsymbol{k}}(W) \subseteq W \cap \boldsymbol{L}^{\boldsymbol{k}}(\boldsymbol{V}) \\
\boldsymbol{L}^{\boldsymbol{k}}[\boldsymbol{V} / W]=\frac{\boldsymbol{L}^{\boldsymbol{k}}(\boldsymbol{V})+W}{W} \\
\frac{\boldsymbol{L}_{\boldsymbol{k}}(\boldsymbol{V})+W}{W} \leqq \boldsymbol{L}_{\boldsymbol{k}}[\boldsymbol{V} / W] \\
\boldsymbol{L}^{\boldsymbol{k}}(\boldsymbol{R}) \boldsymbol{V}=\boldsymbol{L}^{\boldsymbol{k}}(\boldsymbol{V}) \\
\boldsymbol{L}_{\boldsymbol{k}}(\boldsymbol{R}) \boldsymbol{V} \cong \boldsymbol{L}_{\boldsymbol{k}}(\boldsymbol{V}) .
\end{gathered}
$$

The proof of the last lemma is an immediate consequence of parts 2 of Theorems 2 and 4 . In order to show that the inclusion relation in the $b$ parts can be strictly proper, let $\boldsymbol{R}$ be the ring of all $n \times n$ matrices with zeroes above the diagonal, coefficients in any field and with $n$ and $k$ satisfying $0<k \leqq n-2$. In this case the radical $N$ consists of all matrices with zeroes on the diagonal; for any integer $i$ in $0 \leqq i \leqq n, N^{i \perp}=N^{n-i}$ and $\boldsymbol{L}_{i}(R)=\boldsymbol{N}^{n-i}$. To obtain strictly proper inclusions in (1b), (2b), and (3b), simply take $V=N$ and $W=N^{n-1}$.

Conclusions (2a) and (2b) of the last corollary can be rephrased by saying that in the category whose objects are all left $\boldsymbol{R}$-modules $\boldsymbol{V}$ such that $\boldsymbol{R U}=\boldsymbol{U}$ for any submodule $\boldsymbol{U}$ of $\boldsymbol{V}$ and whose maps are module homomorphisms, $\boldsymbol{L}^{\boldsymbol{k}}(\boldsymbol{V})$ is a functor but $\boldsymbol{L}_{\boldsymbol{k}}(\boldsymbol{V})$ is not.

It is not clear what the most natural hypothesis on the ring $\boldsymbol{R}$ and the module $\boldsymbol{V}$ should be in order for the conclusion of the next corollary to be valid.

CoRollary 6. Let $R, N$, and $V$ be as in (1) and (2). In addition let $\boldsymbol{V}$ be a faithful $\boldsymbol{R}$-module. Assume further that for any integer $k=0,1,2, \cdots$ for which $N^{k} \neq\{0\}$, there exists some $x \in \boldsymbol{R}$ such that $N^{k} x \neq\{0\}$ but $N^{k+1} x=\{0\}$. Then if $N$ is nilpotent, the lower Loewy series has a finite number of terms, the last term being $\boldsymbol{V}$. If $\boldsymbol{N}$ is not nilpotent, the lower Loewy series is an infinite ascending chain.

Proof. It suffices to show that if $N^{k} \neq\{0\}$, that then $N^{k \perp} \subset\left(N^{k+1}\right)^{\perp}$. Take $x \in R$ such that $N^{k} x \neq\{0\}$ but $N^{k+1} x=\{0\}$. Then $x \boldsymbol{V} \cong\left(N^{k+1}\right)^{\perp}$, but by the faithfulness of $V, x \boldsymbol{V} \nsubseteq N^{k \perp}$; consequently $N^{k \perp} \subset\left(N^{k+1}\right)^{\perp}$.

It is interesting to note in connection with the next corollary that there is an example of a ring $\boldsymbol{R}$ whose radical consists entirely of nilpotent elements but with $N^{2}=N$, see [2, p. 72] and [7, p. 50].

CoROLlaRY 7. Let $R$ and $V$ be as in (1) and (2) and assume in addition that $\boldsymbol{V}$ is a finitely generated $\boldsymbol{R}$-module. Then the upper Loewy series of $\boldsymbol{V}$ over $\boldsymbol{R}$ either terminates with zero in a finite number of steps or is an infinite descending chain. 
Proof. Under the assumption that the $k$-th term $N^{k} V$ of the upper Loewy series of $\boldsymbol{V}$ is not 0 , it has to be shown that $\boldsymbol{N}^{k+1} \boldsymbol{V} \subset \boldsymbol{N}^{k} \boldsymbol{V}$. It suffices to show, more generally, that for any finitely generated module $W$ over $\boldsymbol{R}, \boldsymbol{N} W \neq W$. If $\boldsymbol{N} W=W$, then the fact that $\boldsymbol{N}$ is a left ideal implies that $W$ is also finitely generated over $N$ and not merely over $\boldsymbol{R}$. But now by [5, p. 200, Proposition 2] we have $N W \neq$ $W$, a contradiction.

EXAMPLES. (i) In sharp contrast to the minimum condition case, the following simple examples show that even if the ring $\boldsymbol{R}$ and module $V$ satisfy conditions (1) and (2), the upper and lower Loewy series need not be of the same length. Let $\boldsymbol{R}$ be the ring of all matrices over any field with an infinite number of rows and columns, with only a finite number of nonzero entries, and having zero entries above the main diagonal. The module $V$ is to consist of column vectors with only a finite number of nonzero components. Then $N$ consists of all matrices with zeroes on the main diagonal. Clearly, $R$ and $V$ satisfy (1) and (2). For any integer $k, N^{k} \boldsymbol{V}$ consists of all column vectors with first $k$ components zero; $\boldsymbol{N}^{k \perp}=\{0\}$ and consequently $\boldsymbol{L}_{\omega}(V)=\boldsymbol{L}_{\omega+1}(V)=0$, where $\omega$ is the first infinite ordinal. Hence the lower series has each term zero whereas the upper series is an infinite properly descending chain with $\boldsymbol{L}^{\omega}(\boldsymbol{V})=\{0\}$. If $\alpha$ is an ordinal which is not a positive integer, then there just does not seem to be in general any way of characterizing the terms $\boldsymbol{L}_{\alpha}(\boldsymbol{V})$ and $\boldsymbol{L}_{\alpha}(\boldsymbol{V})$ of the Loewy series in terms of the radical. In the above example the expected analogue of conclusion 2 in Theorem 2, i.e., $L_{\omega}(V)=\left(\bigcap_{1}^{\infty} N^{k}\right)^{\perp}$, is false.

(ii) An example satisfying (1) and (2) where $\boldsymbol{L}_{\omega}(\boldsymbol{V}) \subset \boldsymbol{L}_{\omega+1}(\boldsymbol{V})=$ $\boldsymbol{V}$ can be constructed by taking $\boldsymbol{R}$ as the ring of all matrices with an infinite number of rows and columns, with zeroes below the main diagonal, with only a finite number of nonzero entries off the main diagonal, and having all the entries on the diagonal eventually constant. The module $\boldsymbol{V}$ is to consist of all column vectors with an infinite number of components but with all the components from some point on eventually constant. The radical $N$ is the set of all matrices with zeroes on the diagonal; $N^{k \perp}$ consists of all vectors having all components zero except possibly the first $k$. Hence $\boldsymbol{L}_{\omega}(V)$ is the subspace of all vectors having only a finite number of nonzero components. Since $V / \boldsymbol{L}_{\omega}(V)$ is isomorphic to the coefficient field, $\boldsymbol{L}_{\omega+1}(\boldsymbol{V})=\boldsymbol{V}$. Although the lower Loewy series is a proper infinite ascending chain, the upper series contains only two distinct terms.

\section{REFERENCES}

1. E. Artin, C. J, Nesbitt, and R. M. Thrall, Rings with minimum condition, University 
of Michigan Press Ann Arbor, 1955.

2. N. Bourbaki, Modules et anneaux semi-simples, Algebre, Chapter 8, Hermann, Paris, 1958.

3. R. Brauer, On sets of matrices with coefficients in a division ring, Trans. Amer, Math. Soc. 49 (1941), 502-548.

4. R. Brauer and E. Weiss, Non-commutative rings, Harvard mimeographed notes (1962).

5. N. Jacobson, Structure of rings, Amer. Math. Soc. Colloq. Pub. 37 (1956).

6. C. E. Rickart, Banach algebras, Van Nostrand, New York, 1960.

7. A. D. Sands, Primitive rings of infinite matrices, Proc. Edin, Math. Soc. (2) 14 (1964), 47-53.

Tulane University

New ORLEans, Louisiana 


\section{PACIFIC JOURNAL OF MATHEMATICS}

\section{EDITORS}

\author{
H. SAMELSON \\ Stanford University \\ Stanford, California \\ R. M. BLUMENTHAL \\ University of Washington \\ Seattle, Washington 98105
}

\author{
*J. DUGUNDJI \\ University of Southern California \\ Los Angeles, California 90007
}

RICHARD ARENS

University of California Los Angeles, California 90024

\section{ASSOCIATE EDITORS}
E. F. BECKENBACH
B. H. NEUMANN
F. WOLF
K. YoSIDA

\section{SUPPORTING INSTITUTIONS}

\author{
UNIVERSITY OF BRITISH COLUMBIA \\ CALIFORNIA INSTITUTE OF TECHNOLOGY \\ UNIVERSITY OF CALIFORNIA \\ MONTANA STATE UNIVERSITY \\ UNIVERSITY OF NEVADA \\ NEW MEXICO STATE UNIVERSITY \\ OREGON STATE UNIVERSITY \\ UNIVERSITY OF OREGON \\ OSAKA UNIVERSITY \\ UNIVERSITY OF SOUTHERN CALIFORNIA
}

\author{
STANFORD UNIVERSITY \\ UNIVERSITY OF TOKYO \\ UNIVERSITY OF UTAH \\ WASHINGTON STATE UNIVERSITY \\ UNIVERSITY OF WASHINGTON \\ * * * * \\ AMERICAN MATHEMATICAL SOCIETY \\ CHEVRON RESEARCH CORPORATION \\ TRW SYSTEMS \\ NAVAL ORDNANCE TEST STATION
}

Mathematical papers intended for publication in the Pacific Journal of Mathematics should be typewritten (double spaced). The first paragraph or two must be capable of being used separately as a synopsis of the entire paper. It should not contain references to the bibliography. Manuscripts may be sent to any one of the four editors. All other communications to the editors should be addressed to the managing editor, Richard Arens at the University of California, Los Angeles, California 90024.

50 reprints per author of each article are furnished free of charge; additional copies may be obtained at cost in multiples of 50 .

The Pacific Journal of Mathematics is published monthly. Effective with Volume 16 the price per volume (3 numbers) is $\$ 8.00$; single issues, $\$ 3.00$. Special price for current issues to individual faculty members of supporting institutions and to individual members of the American Mathematical Society: $\$ 4.00$ per volume; single issues $\$ 1.50$. Back numbers are available.

Subscriptions, orders for back numbers, and changes of address should be sent to Pacific Journal of Mathematics, 103 Highland Boulevard, Berkeley 8, California.

Printed at Kokusai Bunken Insatsusha (International Academic Printing Co., Ltd.), No. 6, 2-chome, Fujimi-cho, Chiyoda-ku, Tokyo, Japan.

\section{PUBLISHED BY PACIFIC JOURNAL OF MATHEMATICS, A NON-PROFIT CORPORATION}

The Supporting Institutions listed above contribute to the cost of publication of this Journal, but they are not owners or publishers and have no responsibility for its content or policies.

* Paul A. White, Acting Editor until J. Dugundji returns. 


\section{Pacific Journal of Mathematics}

\section{Vol. 17, No. 2 \\ February, 1966}

Henry A. Antosiewicz, Boundary value problems for nonlinear ordinary

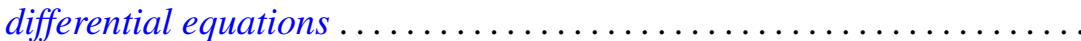

Bernard Werner Levinger and Richard Steven Varga, Minimal Gerschgorin sets. II ........................................

Paul Camion and Alan Jerome Hoffman, On the nonsingularity of complex matrices........................................ 211

J. Chidambaraswamy, Divisibility properties of certain factorials ........ 215

J. Chidambaraswamy, A problem complementary to a problem of Erdös .... 227

John Dauns, Chains of modules with completely reducible quotients ...... 235

Wallace E. Johnson, Existence of half-trajectories in prescribed regions and asymptotic orbital stability ............................ 243

Victor Klee, Paths on polyhedra. II . ........................ 249

Edwin Haena Mookini, Sufficient conditions for an optimal control problem in the calculus of variations ............................ 263

Zane Clinton Motteler, Existence theorems for certain quasi-linear elliptic equations........................................... 279

David Lewis Outcalt, Simple n-associative rings ............... 301

David Joseph Rodabaugh, Some new results on simple algebras ......... 311

Oscar S. Rothaus, Asymptotic properties of groups generation ........... 319

Ernest Edward Shult, Nilpotence of the commutator subgroup in groups admitting fixed point free operator groups .............

William Hall Sills, On absolutely continuous functions and the

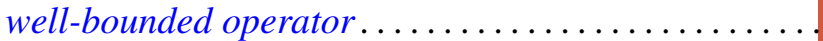

Joseph Gail Stampfli, Which weighted shifts are subnormal ..

Donald Reginald Traylor, Metrizability and completeness in normal Moore spaces 